\title{
The Influence of a Ceramic Recoater Blade on 3D Printing using Direct Metal Laser Sintering
}

Milan Daňa, Ivana Zetková, Pavel Hanzl

Regional Technological Institute, University of West Bohemia - Faculty of Mechanical Engineering, Univerzitní 8, Pilsen 306 14, Czech Republic. E-mail: danam@rti.zcu.cz, zetkova@rti.zcu.cz, hanzlp@rti.zcu.cz

This paper deals with 3D printing of metal parts. There are many principles of 3D printing, for example, DMLS, SLS, CLADDING, etc. This paper is limited to 3D printing using DMLS (Direct Metal Laser Sintering). 3D printing using DMLS can create complex geometries that cannot be created using other metal manufacturing processes. This principle is based on sintering of metal powder in thin layers. Thin layers of powder are applied by a recoater blade. The shape of the part is sintered with a precise and high-wattage laser in this layer. The part is built layer by layer. The printer used for the experimental part of this paper was an EOS M290. The material of the recoater blade was ceramic and the powder material was MS1. There are several types of recoater blade but this article focuses on the limitations of using a ceramic blade recoater. The influence of the orientation and size of support structures depending on the recoater blade was examined. Also, the effect of the height of the support structures on their vibrations was investigated and the vibration of the support structures was described in detail. The influences and limitations of the recoater blade were investigated.

Keywords: 3D print, DMLS, MS1 - 1.2709, recoater blade, limitations

\section{Introduction}

Additive manufacturing (AM) is a very dynamically developing manufacturing technology and this means that development is focusing on specific areas of the process. Additive manufacturing is a very large area with a lot of principles and methods and therefore this paper is limited to DMLS (Direct Metal Laser Sintering). This method is based on the application of thin layers of powder, which are sintered by a laser beam. The part is cut into individual slices, while the distance of individual cuts depends on the thickness of the applied layer of the powder. The shape of the part is sintered using a laser beam in each layer. The parts are built layer by layer. The principle of DMLS allows printing of various metallic materials such as maraging steel, Inconel 718, titanium, aluminium, etc. The technology can solve various issues regarding the manufacturability of parts, but there are cases when it is not possible to create a part using this method. Even this technology has its limitations, which can be split between those arising from the principle of the technology, and geometric limitations. This paper is focused on the limitations arising from the principle of the technology and especially the application of powder during the printing process. The following figure shows the principle of applying powder during the printing process.

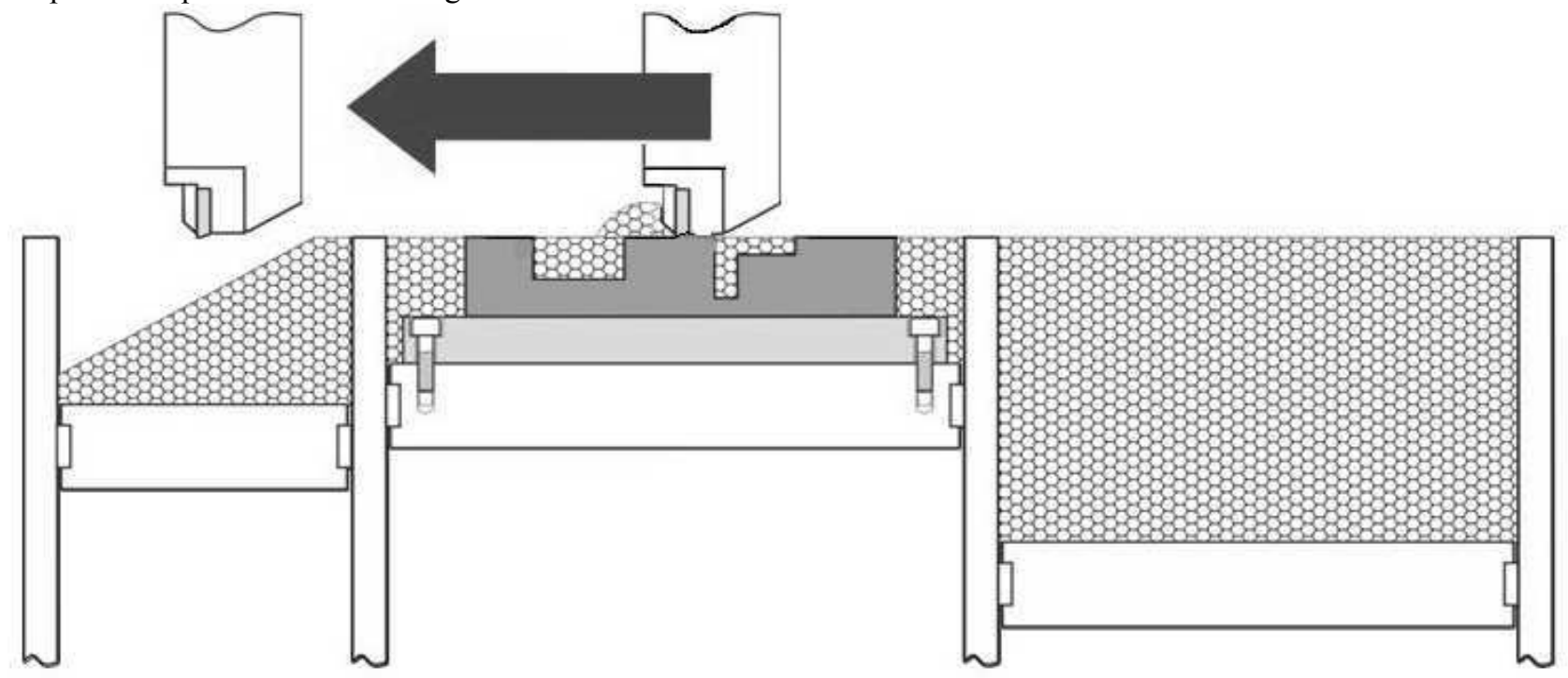

Fig. 1 Principle of applying powder [1]

The application process seems very simple and almost trivial, but that is not quite true. The recoater blade has to ensure the correct application of powder in a compact layer. The material of the recoater blade can have a large influence on the printing process and applying the powder layer. It can be assumed that the ceramic recoater blade will create some problems during the printing process. This is why the experiment focused on a ceramic recoater blade and its influence on this process. One of the main goals of this paper is to find the limitations resulting from using a ceramic recoater blade. The main aspects and limitations of this recoater blade will be described in this 
article. Reference [1] deals extensively with the descriptions of basic principles and also details. Reference [2] gives information about the good orientation of the thin walls of the parts depending on the recoater blade. This reference describes the individual positions of the parts and their propensity to contact with the blade recoat and the propensity to vibrations. Reference [3] provides information about the metallography of the printed steel 1.2709. The information from this reference is useful for cognition of the printed material. Reference [4] is also focused on 3D printing and especially on the vibrations of the recoater blade. The author here measures the acceleration of the recoater blade caused by contact with the part or the support structure. Reference [5] gives information about the dependence of the orientation of the thin walls on the recoater blade. If the orientation is poor, problems occur during printing.

From the searched sources it follows that the applying element has a considerable influence on applying the powder layer, vibrations of support structures and parts, safety of printing and many other things.

\section{Experimental device and adjustment}

\subsection{Machine and parameters}

The printer used for the experimental part of this paper was an EOS M290. This printer works on the principle of DMLS (Direct Metal Laser Sintering). The M290 has a building volume of $250 \times 250 \times 325 \mathrm{~mm}$, laser power $400 \mathrm{~W}$ and scan speed up to $7 \mathrm{~m} / \mathrm{s}$. The experimental print was started with the process parameters as recommended by the manufacturer. The default process parameters used are EOS_Direct_Part for the test samples with support structures, and EOS_Direct_tools for the test samples without support structures. The support structures have special parameters named EOS_Support_Structures. Individual parameters such as laser power, scan speed and many others are included under these designations. For example, the parameters for printing the part are laser power $285 \mathrm{~W}$, Laser speed $960 \mathrm{~mm} / \mathrm{s}$, Hatch $0.11 \mathrm{~mm}$, etc. But these parameters have a different setting depending on their position in the part. The skin of the part has different parameters than the core of the part. The layer thickness was 40 micrometres. [6]

\subsection{Recoater blade}

This printer can use three types of recoater blades. The first type of recoater blade is ceramic $\mathrm{ZrO} 2$, the second one is high speed steel and the third one is a carbon fibre brush. All these recoater blade materials have different properties. The ceramic recoater blade was used for printing from maraging steel as recommended by the manufacturer. The basic properties of ceramic are high rigidity, dimensional and temperature stability, chemical resistance, etc. A huge advantage is its long life. The main disadvantage is its intractability and therefore the propensity to hard contact of the blade with the printed part. This material has a tendency to break away during the hard contact. In this case of chipping, a 'wave' is created on the powder layer. This causes a thicker layer of powder to be used in the area of the chip. If the 'wave' is located on the printed part it is necessary to use a new recoater blade edge. Blades made from ceramic and high speed steel have two usable edges. The geometry of the blades is different. EOS has patented the geometries of these recoater blades for many years. The geometry of a ceramic recoater blade is shown in Fig. 2.

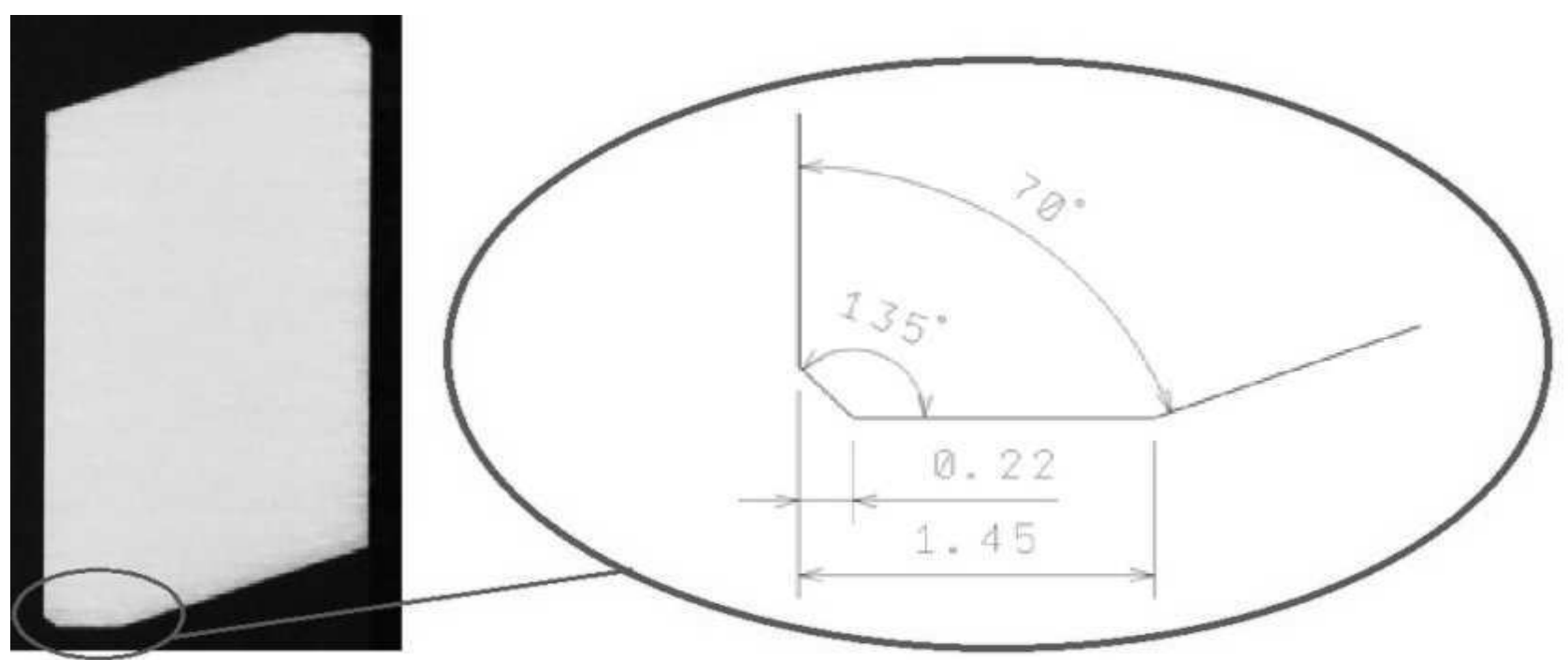

Fig. 2 Geometry of ceramic recoater blade-detail

The recoater blade has to be longer than the building platform to be able to apply powder at one time. If the recoater blade is divided in several parts it could lead to unequal application of powder and a different depths of powder layers could arise on the platform. Transitions between individual parts of the recoater blade may cause problems with powder application. The recoater blade has the task of applying a compact, thin powder layer. When applying the powder, an 'avalanche effect' could occur, causing dirt and burns to be pushed away to the collector duct. This effect is conditional on applying a larger amount of powder than is strictly necessary. Another reason for the application of a larger amount of powder is that the powder volume is not the same as the volume of 
the sintered material. 'Depending on the material, the powder density is approximately 50\% lower compared to solid. Due to solidification and increase in density, the Xthickness (approximately 1/2 of layer thickness) is added to the nominal layer thickness.' [10]

\subsection{Reasons for contact}

The recoater blade edge applies a thin layer of powder on the building platform during the printing. In some cases, there have been problems with the contact between the recoater blades and the printed parts or the support structures. There may be several reasons for the contact between the recoater blade and the parts or support structures. A very common reason for the contact of the recoater blade is dirt, burns and protrusions. These items are created on the top of the printed surfaces. The following figure shows clearly the burns that protrude above the printed surface. Another reason for contact is the separation of the printed parts from the support structures during printing. The deformation of the part from internal stress can be another reason for contact.

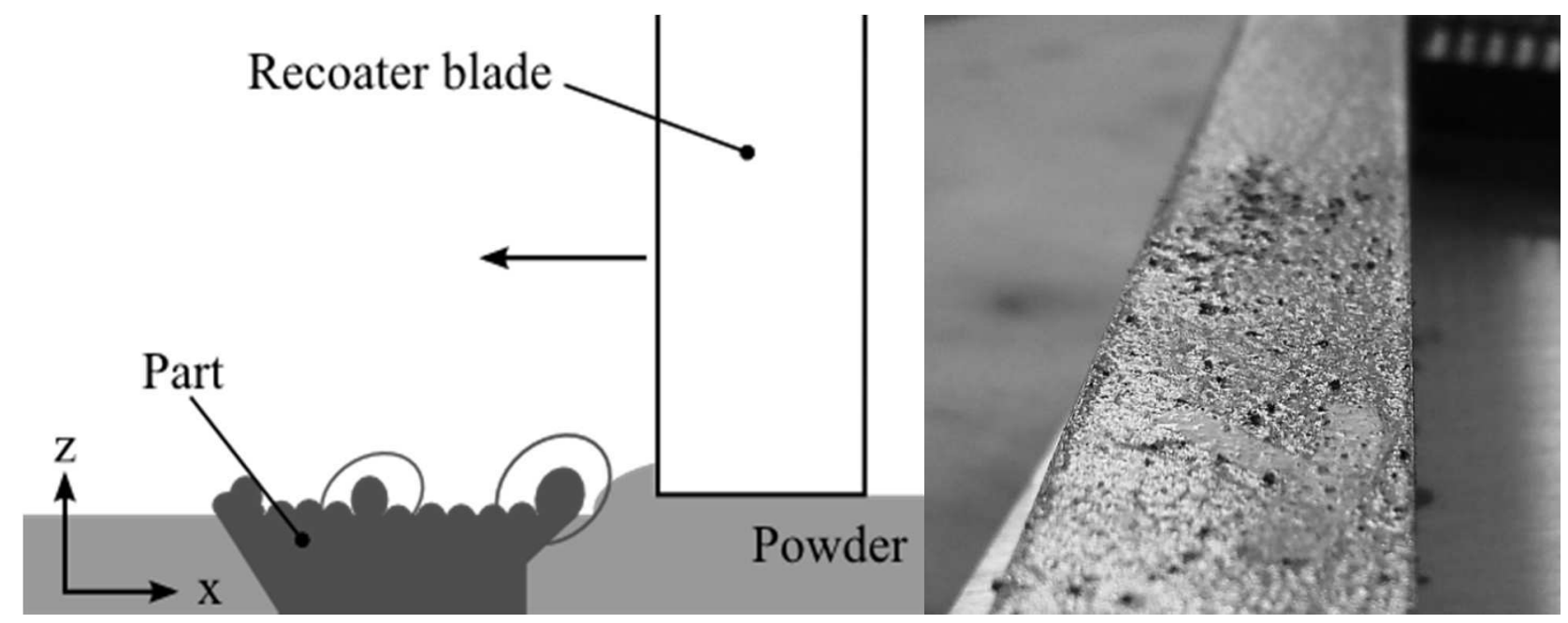

Fig. 3 Protrusions over the application of a new layer [7]

\subsection{Orientation of support structures and parts}

The contact between the recoater blade and support structures leads to their vibrations. Almost all types of support structures are susceptible to vibrations, nevertheless the structure type 'Volume' is more durable than others. The shape and size of support structures have a great influence on this vibration. If the support structure is rectangular, measuring $70 \times 70 \mathrm{~mm}$, it will be less susceptible to vibration than a rectangle with dimensions $10 \times 30 \mathrm{~mm}$. The height of the support structures also has a great impact. The higher the support, the more prone it is to vibrations. Nevertheless, the orientation of support structures on the building platform has a huge impact. It is suitable to place the support structures with respect to the recoater blade. If the support structure is oriented to the weak side, perpendicular to the recoater blade, it will be more prone to vibrations. This case is shown in the diagram marked number 1 in Fig. 4 (left). If the support structures are situated as shown by number 2 in the diagram, then the support structure is stronger and can resist vibrations. This is due to the fact that the leading edge is smaller and the support structure has better stability. This phenomenon is not only typical for the support structures, but printed parts are also prone to vibration. The right part of Fig. 4 shows problems during the printing process due to the wrong orientation of the printed part. If the thin portions of the part are oriented perpendicularly to the blade, then the part is printed without any problems.

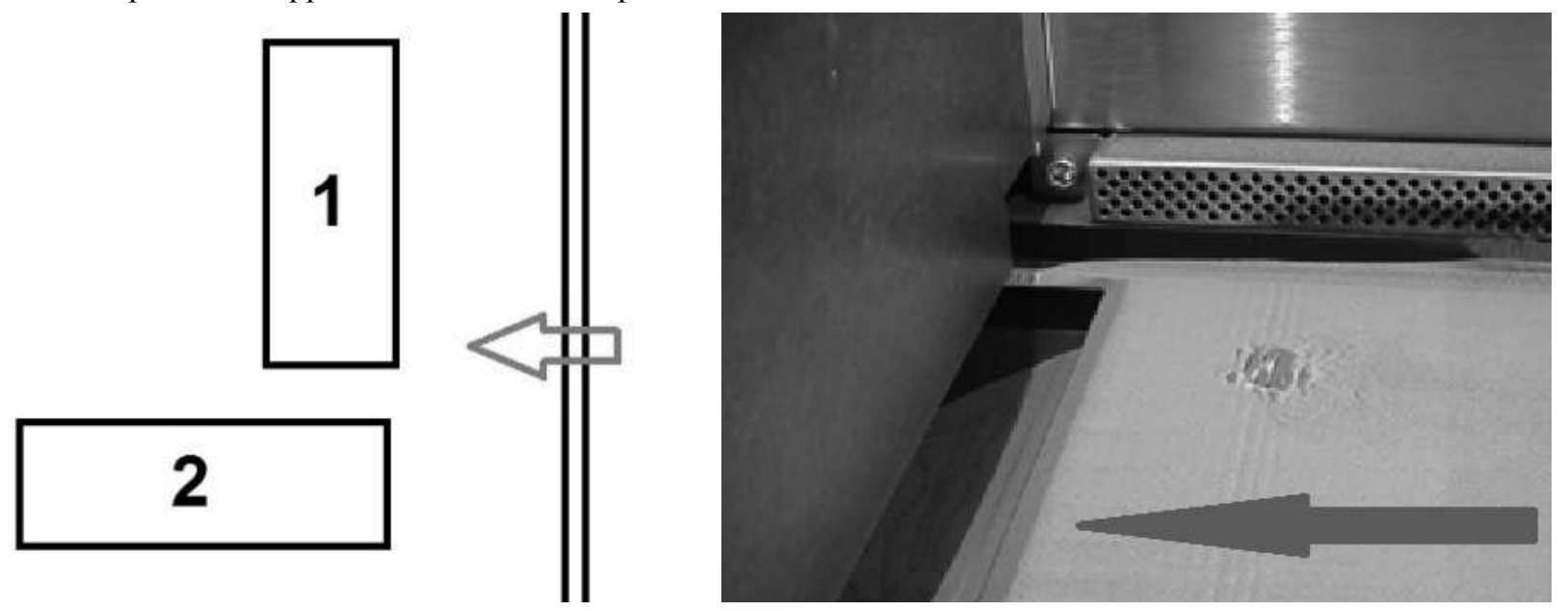

Fig. 4 Position of support structures depending on recoater blade (left), and problems during the print (right) [5] 


\subsection{Vibrations process}

The following figure describes how vibrations arise and the whole vibration process. Sector one shows the sintering of the powder. In this case, there are some problems such as protrusions, dirt or degradation of the walls due to internal stress, etc. The second sector shows contact between the blade and the printed part. The part is pushed by the recoater blade and the vibrations occur when the contact is interrupted. The powder that is near the part is pushed away due to vibrations of the part, as can be seen in the third sector. Then (sector 4) the sintering occurs but the powder is missing on the top of the part. The previous layer is re-sintered. The two layers are sintered in the next step of the process if vibration of the support does not occur again. This is a problem, but a much more serious problem can be caused by the powder that has been relocated during vibrations. This case is shown in the fifth sector. There can be a sintering of an uncontrolled amount of powder. In this case, it is almost certain that contact between the recoater blade and this sintered material will occur and it will cause additional vibrations. In some cases, it may lead to melting of a large layer of powdery material so that a big protrusion is created. This protrusion can cause interruption of the recoater blade during the application process. In an extreme case, the protrusion may be so large that it can cause recoater blade interruption during the backward movement.

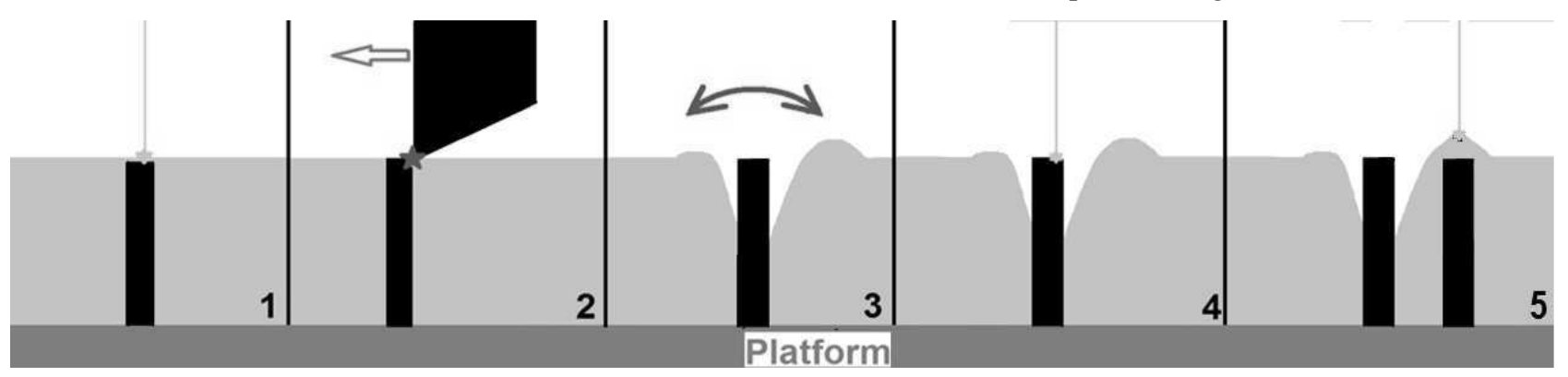

Fig. 5 Procedure for creating vibrations of support structures and their consequences

\section{Printing problems caused by recoater blade}

As already mentioned above, the vibrations of the support structure are caused by contact between the recoater blade and the support structure, but in extreme cases, the support structure may be deformed or broken away.

Deformation of the support structures can cause significant complications. These complications result from the missing part of a support structure or an entire support structure in the place that needs to be supported. If the support structure is missing, it can cause several problems, for example: building newly created islands in the powder bed, poor surface quality on the part in this place, very bad heat transfer from this area, worsening the anchoring of the part or the newly created islands and many other things. In most cases these problems cause defects on the printed parts. To these defects can be added, for example: missing pieces of the part, poor geometry of parts, poor surface quality and many other things. In some cases, defects can cause much more serious problems. In these cases, the safety of the printing process may be compromised. It is recommended to deactivate the printing of a part and then it is possible to complete the printing of other parts on the building platform.

If the print process stops due to problems with printing the part and then the printing process is started from the next layer, it is not a good idea in most cases, because the print will be stopped for several following layers. The print will probably be stopped, because the causes of the print interruption have not been removed. In this case, it is necessary to stop the printing process and rebuild the support structures or modify the parts in an appropriate way.

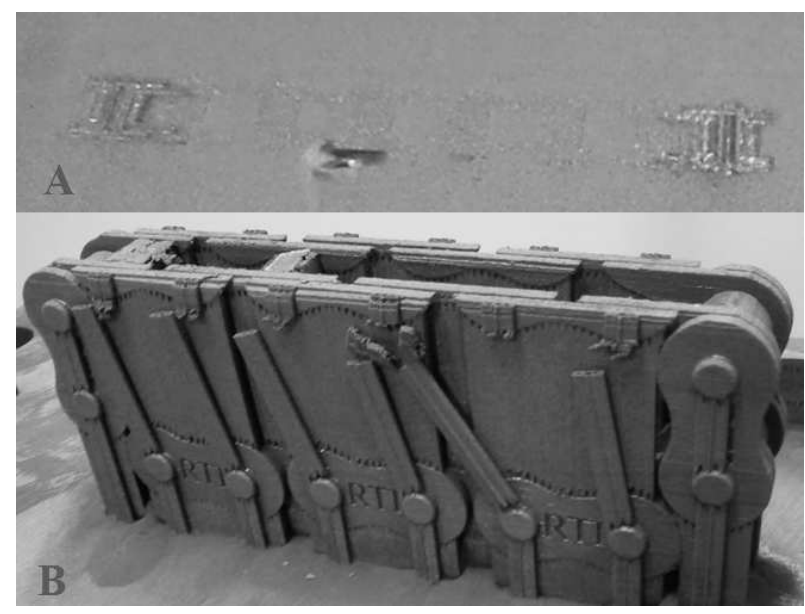

Fig. 6 Deformation of support structures - A) During the print, B) After the print

The first problem was caused by poor orientation of the thin walls in relation to the recoater blade. The first sample A was oriented perpendicularly to the recoater blade. In this case, there were no deformations. However, sample B was oriented parallel to the recoater blade and that was a problem. The recoater blade bent the thin wall, but the print continued at the same location. The print continued for several dozen layers but the problems escalated. The safety of the printing was threatened because this part had to be omitted. In contrast, sample A was printed completely without any problems. 

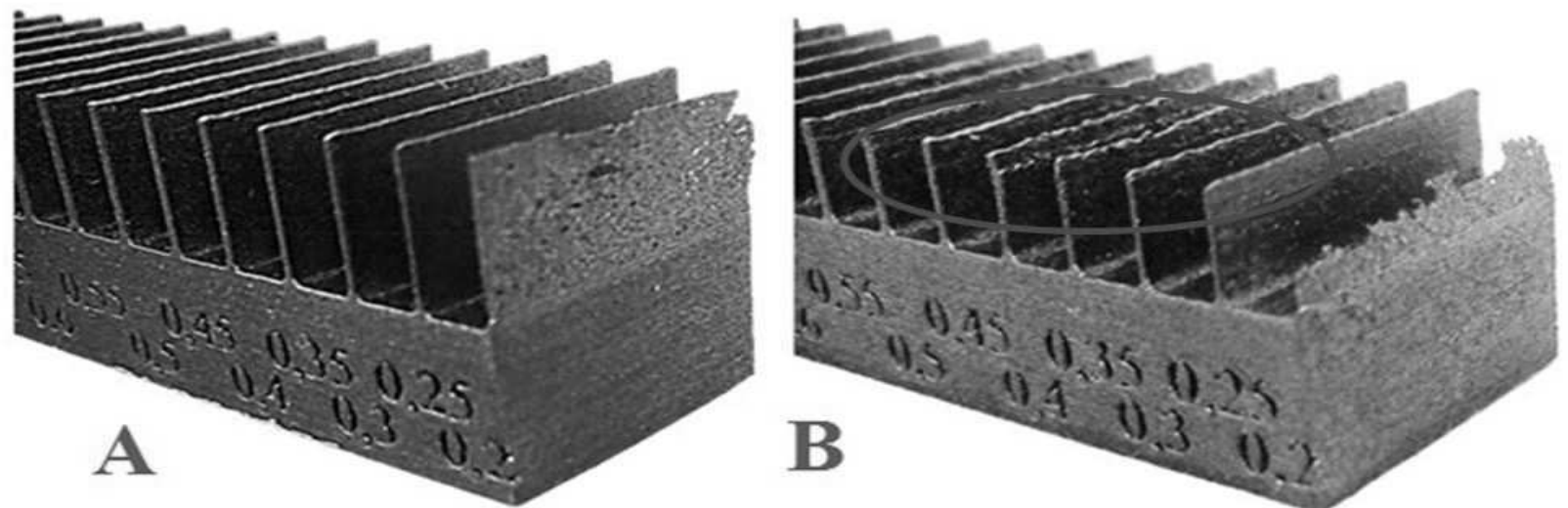

Fig. 7 Comparison of different orientations of the same part-A-positon 2, B-position 1[5]
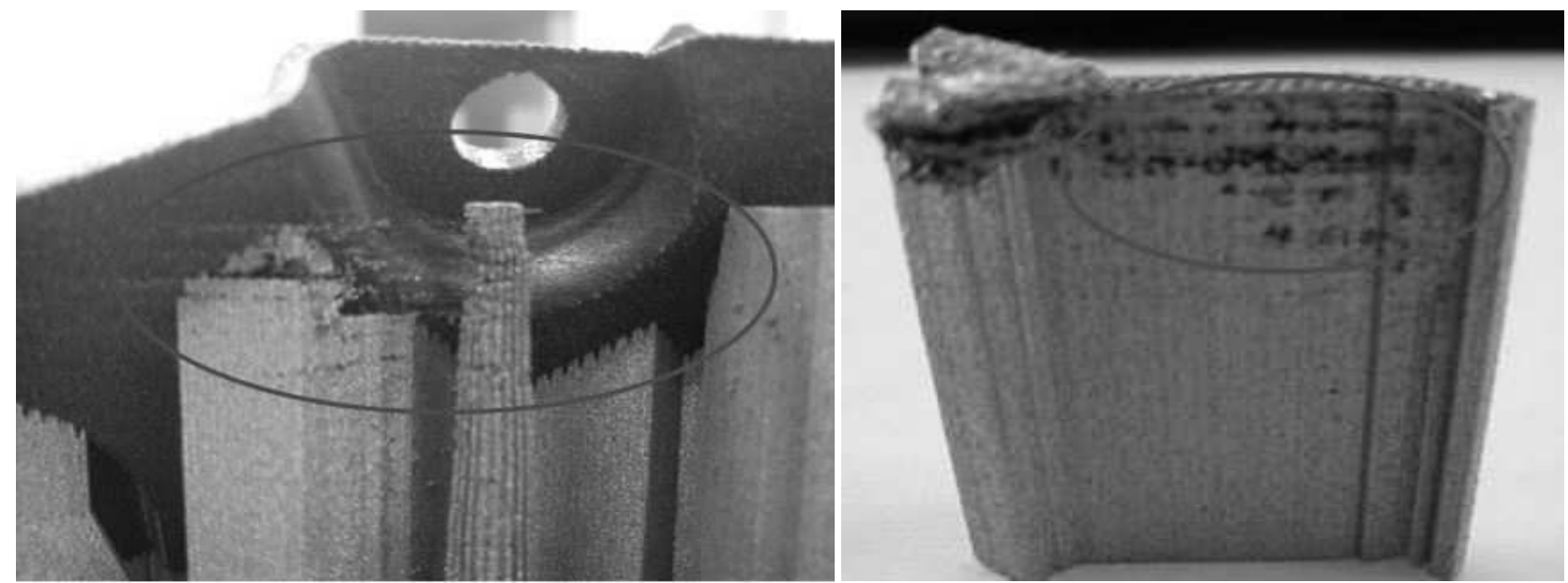

Fig. 8 The consequences of vibrations of support structures

The vibration of the part or support structures can be detected even after printing because of their consequences, which are easily identifiable. If the powder is missing in several layers and the laser melts only the same printed layer at the top of the printed part then the printed part can be porous. Cavities are created due to the fact that the laser is able to melt approximately three layers. If the layer is larger than 4 layers, then an interface may occur between the part and the new printed layer. This interface can be created due to imperfect connection between the melted powders and the printed part. These areas are torn off from the part during the contact with the recoater blade. The following picture shows the porosity of the support structures.

\section{Different recoater blade}

The next figure represents a printed part using a comparative printer with a different type of recoater blade. The basic dimensions of the test cube are $75 \times 75 \times 75$ $\mathrm{mm}$. The cube is supported using very thin and very high support structures. Some of the support structures are perforated. The cross-section of some of the support structures is approximately $3 \times 5 \mathrm{~mm}$ with a height up to 100 $\mathrm{mm}$. The orientation of the support structures is random to the recoater blade. The connecting teeth are situated between the building platform and the support structures. This setting of the support structures reduces the bond strength of the connection and increases the susceptibility to damage. A flexible recoater blade was used during the print, making it possible to print these support structures. If a flexible recoater blade is used, then there is no hard contact between the recoater blade and the support structures, meaning that there are no vibrations or other problems resulting from vibrations. These support structures cannot be printed using a ceramic recoater blade. [9]

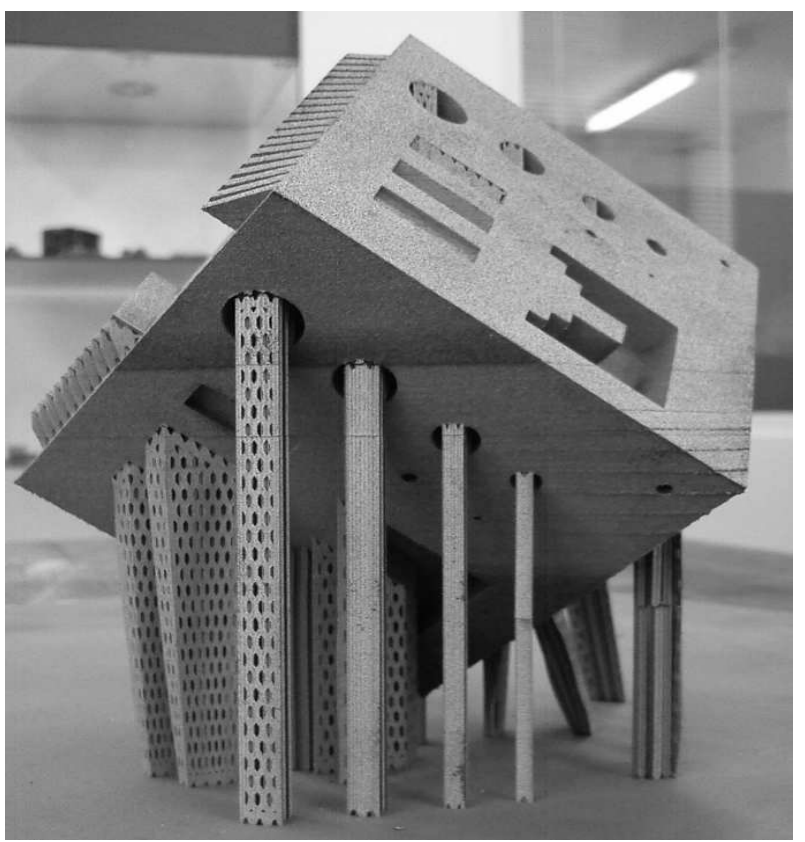

Fig. 9 Support structures printed with a flexible recoater blade [8] 


\section{Conclusion}

This paper presents the effects of using a ceramic recoater blade on the $3 \mathrm{D}$ printing process. These influences were identified through several experiments and are also a result of the day-to-day use of the printer. The use of a ceramic recoater blade has several limitations and also depends on the strength and orientations of the support structures or parts. The rigidity of the support structure depends on its size, orientation and especially on its height. If the support structure is insufficiently rigid, it may cause vibrations to arise due to contact between the recoater blade and the support structure. The orientation of the part or support structures also has a considerable effect on vibration. Vibration generation and the procedure of vibration are described in detail in this article. Using a ceramic recoater causes several problems that can be reflected in the quality of the printed parts. The vibrations of the part or support structures can be detected even after printing due to their consequences, as shown in the figures in section 3 of this paper. The support structures should be created with respect to the type of recoater blade used. If a support structure is not very strong, it is not advisable to select a parallel orientation to the recoater blade.

In the experimental part of this paper it was concluded that the material of the recoater blade has a very large influence on the printing process, especially on the vibration of the support structures and the safety of the print. From the experiments that were carried out it is not possible to set precise limits for when it is not suitable to use the ceramic recoater blade. In some cases, it is not possible to print a part using a ceramic recoater blade, as shown in Fig. 9. Therefore it is necessary to use a different type of recoater blade. A flexible recoater blade was used for a comparative experiment. This type of recoater blade allows printing without hard contact between the part and the recoater blade. Vibrations do not arise as a result. Using this type of recoater blade opens up new printing possibilities.

\section{Acknowledgement}

\section{This paper is based upon work sponsored by project SGS-2016-005.}

\section{References}

[1] Eos training materials. Internal documents.

[2] H. KRAUSS and M. ZAEH, "Investigations on Manufacturability and Process Reliability of Selective Laser Melting," Physics Procedia, vol. 41, no. 0, pp. 815 - 822, 2013, Lasers in Manufacturing (LiM 2013). [Online]. Available: http://www.sciencedirect.com/science/article/pii/S1875389213001673

[3] KUCEROVA, L. ZETKOVA, I. (2016). Metallography of 3D Printed 1.2709 Tool Steel. In MANUFACTURING TECHNOLOGY. February 2016, Vol. 16, No.1. s. 140 -144. ISSN 1213-2489

[4] REINARZ and G. WITT (2012). "Process Monitoring in the Laser Beam Melting Process - Reduction of Process Breakdowns and Defective Parts," in Proceedings of Materials Science \& Technology 2012, 2012.

[5] DANA, M. ZETKOVA, I. HANZL, P. (2016). Prining of Thin Walls using DMLS. In MANUFACTURING TECHNOLOGY. October 2016, Vol. 16, No.4. s. 883 -896. ISSN 1213-2489

[6] EOS M290. EOS. [online]. [cit. 2017-02-10]. Available from: https://cdn2.scrvt.com/eos/public/413c861f2843b377/93ef12304097fd70c8663 44575a4af31/EOS_System-DataSheet-EOSM290.pdf

[7] J. JACOBSMÜHLEN, S. KLESZCZYNSKI, G. WITT, and D. MERHOF. (2015). Elevated Region Area Measurement for Quantitative Analysis of Laser Beam Melting Process Stability. Available from: https://sffsymposium.engr.utexas.edu/sites/default/files/2015/2015-44-zurJacobsmuehlen.pdf

[8] ZETKOVA, I. ZETEK, M. DANA, M. KUTLWASER, J. (2016). Critical Parameters of Metal Laser Sintering with its Impact on Geometrical Accuracy of a Component. In MANUFACTURING TECHNOLOGY. October 2016, Vol. 16, No.4. s. 1216 -1222. ISSN 12132489

[9] CAPKOVÁ, V. (2015). Restrictive parameters of 3D printing .Pilsen 2015. Thesis. SUPERVISOR Ing. Zetek, Ph.D, Available from: https://otik.uk.zcu.cz/handle/11025/9318

[10] SRIVATSAN, T. (ED.), SUDARSHAN, T. (Ed.). (2015). Additive Manufacturing. ISBN 9781498714785. Boca Raton: CRC Press. 